\title{
Dy, Er, and Yb isotope systematics in meteorites and their components
}

\author{
Q.R. SHOLLENBERGER ${ }^{1}$, G.A. BRENNECKA ${ }^{2}$
}

${ }^{1}$ University of Münster, Germany (*shollenberger@wwu.de) ${ }^{2}$ Lawrence Livermore National Laboratory, Livermore, USA

Investigating numerous isotope signatures in primitive meteorites and their components can provide important information about stellar processes and early Solar System reservoirs. For example, comparing the isotopic compositions of calcium-aluminum-rich inclusions (CAIs), the first solids to condense in the protoplanetary disk, to later formed bulk meteorites can inform researchers about how reservoirs in the protoplanetary disk evolved over time [e.g., 1]. For many elements, normal (non-FUN) CAIs have uniform and distinct isotopic anomalies relative to terrestrial and bulk meteorite compositions that can be explained by excesses/deficits in material produced by the $r$-process of nucleosynthesis [2-4]. In contrast, previous studies of bulk chondrites [e.g., 4-6], and studies that utilized step-wise leaching of primitive chondrites [e.g., 7] have shown that isotopic anomalies in bulk meteorites are best attributed to variable contributions of material produced by the $s$-process of nucleosynthesis. Whereas many elements have been studied in both CAIs and bulk meteorites, integrated information for heavier isotopic systems $(A>154)$ is currently lacking.

To address this issue, we present $\mathrm{Dy}, \mathrm{Er}$, and $\mathrm{Yb}$ isotope data for CAIs from multiple parent bodies, bulk meteorites from a variety of classes and groups, as well as step-wise acid leachates from the Murchison meteorite $[3,8]$. In this presentation, I will summarize the $\mathrm{Dy}, \mathrm{Er}$, and $\mathrm{Yb}$ isotope data from CAIs, bulk meteorites, and Murchison acid leachates and describe how these objects relate to one another and to the evolution of the early Solar System. Overall, this integrated dataset demonstrates there is a progressive homogenization of $\mathrm{Dy}, \mathrm{Er}$, and $\mathrm{Yb}$ isotopes through time recorded in the meteoritic record.

References: [1] Burkhardt et al. (2019) GCA, 261, 145. [2] Brennecka et al. (2013) PNAS, 110, 17241. [3] Shollenberger et al. (2018) EPSL, 495, 12. [4] Burkhardt et al. (2011) EPSL, 312, 390. [5] Fischer-Gödde et al. (2015) GCA, 168, 151. [6] Bouvier \& Boyet (2016) Nature, 537, 399. [7] Qin et al. (2011) GCA, 75, 7806. [8] Shollenberger \& Brennecka (2020) EPSL, 529. 